\title{
Machine Learning and the Meaning of Equal Treatment
}

\author{
Josh Simons ${ }^{1}$, Sophia Adams Bhatti ${ }^{2}$, Adrian Weller ${ }^{3,4}$ \\ ${ }^{1}$ Harvard University, ${ }^{2}$ Wavelength Law, ${ }^{3}$ University of Cambridge, ${ }^{4}$ The Alan Turing Institute
}

\begin{abstract}
Approaches to non-discrimination are generally informed by two principles: striving for equality of treatment, and advancing various notions of equality of outcome. We consider when and why there are trade-offs in machine learning between respecting formalistic interpretations of equal treatment and advancing equality of outcome. Exploring a hypothetical discrimination suit against Facebook, we argue that interpretations of equal treatment which require blindness to difference may constrain how machine learning can be deployed to advance equality of outcome. When machine learning models predict outcomes that are unevenly distributed across racial groups, using those models to advance racial justice will often require deliberately taking race into account.

We then explore the normative stakes of this tension. We describe three pragmatic policy options underpinned by distinct interpretations and applications of equal treatment. A status quo approach insists on blindness to difference, permitting the design of machine learning models that compound existing patterns of disadvantage. An industry-led approach would specify a narrow set of domains in which institutions were permitted to use protected characteristics to actively reduce inequalities of outcome. A government-led approach would impose positive duties that require institutions to consider how best to advance equality of outcomes and permit the use of protected characteristics to achieve that goal. We argue that while machine learning offers significant possibilities for advancing racial justice and outcome-based equality, harnessing those possibilities will require a shift in the normative commitments that underpin the interpretation and application of equal treatment in non-discrimination law and the governance of machine learning.
\end{abstract}

\section{CCS CONCEPTS}

- Applied computing $\rightarrow$ Law.

\section{KEYWORDS}

equal treatment, fairness, philosophy, politics, machine learning

\section{ACM Reference Format:}

Josh Simons ${ }^{1}$, Sophia Adams Bhatti ${ }^{2}$, Adrian Weller ${ }^{3,4}$. 2021. Machine Learning and the Meaning of Equal Treatment . In Proceedings of the 2021 AAAL/ACM Conference on AI, Ethics, and Society (AIES '21), May 19-21, 2021, Virtual Event, USA. ACM, New York, NY, USA, 11 pages. https://doi.org/10.1145/ 3461702.3462556

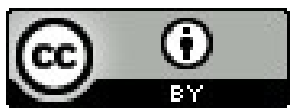

This work is licensed under a Creative Commons Attribution International 4.0 License.

AIES '21, May 19-21, 2021, Virtual Event, USA.

(C) 2021 Copyright held by the owner/author(s).

ACM ISBN 978-1-4503-8473-5/21/05.

https://doi.org/10.1145/3461702.3462556

\section{INTRODUCTION}

Approaches to non-discrimination are generally informed by two principles: striving for equality of treatment and advancing various notions of equality of outcome. This paper considers when and why there are trade-offs in the design of machine learning models between respecting formalistic interpretations of equal treatment and advancing equality of outcome. We describe formalistic interpretations of equal treatment that require blindness to difference, including differences identified by protected characteristics, as the treatment-as-blindness approach. Applications of discrimination law motivated by treatment-as-blindness will often prohibit design choices in machine learning that strive to advance equality of outcomes.

We use the a hypothetical discrimination suit against Facebook to explore cases in which well-calibrated machine learning models trained on representative datasets predict outcomes that are unevenly distributed across protected groups [8, 48]. In such cases, many approaches that advance equality of outcome may violate equal treatment-as-blindness, because, as critical scholars of race and gender have persuasively argued, advancing racial and gender equality will often require deliberately taking race and gender into account $[30,45,46,87]$. We explore the scope of this problem, specifying the conditions under which there may be a choice in designing and deploying machine learning models between respecting equal treatment-as-blindness and advancing equal outcomes.

We then explore the normative stakes of this tension. Many technical approaches aim to find methods for training and deploying machine learning systems that enforce some notion of equality of outcome while respecting equal treatment-as-blindness. While such methods exist and may sometimes be useful, such as disparate learning processes (DLPs), they are likely to be sub-optimal on several dimensions, and more importantly, technical methods cannot address the underlying philosophical problem of how to understand and apply the principle of equal treatment. Instead we describe three pragmatic policy options underpinned by distinct interpretations and applications of equal treatment: status quo, industry-led, and government-led approaches.

In jurisdictions like the US and UK where formalistic interpretations of equal treatment often motivate the application of discrimination law, the use of protected characteristics in decision-making is generally prohibited, with a narrow range of targeted exemptions $[18,35,89]$. In such cases, the status quo may prevent organisations from exploring how to use machine learning to reduce persistent inequalities of outcome. This status quo is not neutral.

The industry-led approach would permit institutions to actively reduce inequalities of outcome by using protected characteristics in a narrow set of domains, requiring greater transparency and accountability about how and why those characteristics are used. This would require a shift in normative interpretations of equal treatment, away from blindness to difference and towards awareness and sensitivity to difference. We advocate for such a shift and 
suggest that the increasing richness and availability of data offers opportunities to be more discerning about who is similar to whom across the multiple, connected dimensions of disadvantage $[9,81]$.

Finally, the government-led approach would impose positive duties on institutions to consider how best to advance equality of outcomes in a defined set of decision contexts and permit the use of protected characteristics to achieve that goal. In contexts where positive duties apply, institutions would be able to rebut accusations that a system violates equal treatment-as-blindness by demonstrating they undertook reasonable measures to advance equality of outcome. These positive duties would shift the focus and enforcement of equality and civil rights law from ex post demonstrations of individual harm to ex ante duties to advance equality. Such duties could be especially salient in ensuring technology companies with monopoly power build and use machine learning to advance racial justice, gender equality, and socio-economic opportunity.

While we are not the first to explore the meaning of the principle of equal treatment, our contributions to recent work are to show that (1) longstanding tensions in civil rights and equality law are rooted in an older philosophical debate about the meaning of equal treatment; (2) these tensions are a pressing practical concern for civil rights regulators and technology companies; (3) addressing them is not primarily a technical challenge but a philosophical question and policy choice about when, how, and why equal treatment should be interpreted and applied; and (4) to evaluate high-level policy frameworks that would support rather than constrain organisations seeking to develop technical innovations to reduce enduring inequalities of outcome.

\section{CONTEXT}

Aristotle was the first to write down the principle that like cases should be treated similarly and unlike cases dissimilarly, and more ambitiously, that unlike cases should be treated "in proportion to their unlikeness" [10, 26, 82]. A few hundred years earlier, Aesop's fable told of a fox who invites a crane for dinner, then serves soup in a shallow dish. The fox overlooks a relevant difference - the crane has a long beak - which requires differential treatment - they need different vessels to drink from. The crane makes the point by inviting the fox for dinner and serving soup in a long, narrow jar.

In itself, the principle of equal treatment is simply a formal relationship that lacks substantive content. Aristotle did not believe there was any way to deduce the meaning of equal treatment from first principles, but rather, that disagreements about similarity and difference are central to debates about justice and the best political regime. Living together in political societies requires us to justify judgements about who is similar and different to whom in particular cases. Instead of attempting to find a single, universal answer to the question of who is similar to whom, the essence of living as equal citizens involves debating and justifying the meaning we give to the principle of equal treatment: who should be considered similar to whom and what kinds of differences justify differential treatment across different contexts [10,38].

In many countries, equal treatment has come to mean some form of blindness, often in practice, blindness to protected characteristics. Decision-making systems that respect equal treatment must ensure people are treated without regard to morally irrelevant differences like race and gender $[72,86,90]$. The basic liberal response to racial injustice, for instance, has been to insist that since race is morally irrelevant to the distribution of social benefits and burdens, decisions should not be made because of and should be blind to race. "The way to stop discrimination on the basis of race," argued John Roberts, the Chief Justice of the US Supreme Court, "is to stop discriminating on the basis of race" [79].

This is not the only way equal treatment can be interpreted. "The opposite of 'racist' isn't 'not racist'," argues Ibram X. Kendi in a book widely cited by Black Lives Matter activists following the murder of George Floyd, "it is 'antiracist" [52]. Or as Beverly Tatum put it, "visualize the ongoing cycle of racism as a moving walkway at the airport." The effort to be blind is like "standing still on the walkway. No overt effort is being made, but the conveyor belt moves the bystanders along to the same destination." The only way to change your destination is to "turn around" and walk "actively in the opposite direction at a speed faster than the conveyor belt" [91]. On this view, people of different races and genders are unlike in precisely the sense that justifies differential treatment - that is why those characteristics are protected [12, 63, 85]. "The way to stop discrimination on the basis of race," rebuffed Justice Sonia Sotomayor, "is to speak openly and candidly on the subject of race, and to apply the Constitution with eyes open to the unfortunate effects of centuries of racial discrimination" [89].

This treatment-as-awareness view was most prominently articulated during the civil rights movement. In 1976, Owen Fiss, the legal scholar, criticised treatment-as-blindness because it "does not formally acknowledge social groups, such as blacks; nor does it offer any special dispensation for conduct that benefits a disadvantaged group. It only knows criteria or classifications; and the color black is as much a racial criterion as the colour white." It treats positive action as "a form of discrimination" that "is equally arbitrary since it is based on race," and as such, provides no "basis or standards for determining what is 'reform' and what is 'regression'” [33]. As Justice Henry Blackmun put it in 1978: "in order to get beyond racism, we must first take account of race" [22].

Countries that have confronted racial and gender oppression through their own democratic institutions often interpret equal treatment to explicitly require differential treatment on the basis of differences produced by past inequalities. For instance, the concept of equality in the Constitution of South Africa, which was deliberately written to confront the a violent history of racial oppression, "includes... remedial or restitutionary equality. Such measures are not in themselves a deviation from, or invasive of, the right to equality guaranteed by the Constitution. They are not 'reverse discrimination' or 'positive discrimination," exceptions to the general principle that equal treatment means blindness, but rather, "they are integral to the reach of equality protection" [7].

Machine learning may invite a confrontation between these two accounts of equal treatment. Machine learning models often reveal the many ways in which people of different races and genders are in fact different in precisely the sense that justifies differential treatment. Addressing enduring unjustified inequalities will require the regulation of decision-making to permit, encourage, and even require awareness and sensitivity to difference, rather than imposing blindness to it, including differences identified by protected traits like race and gender [32,64]. 


\section{RELATED WORK}

Debates about the meaning of equal treatment stretch back to Aristotle. Aristotle's political philosophy "aims to show how a certain equality" can be "achieved among different people who have different characteristics, possess different things, and live through different experiences" [10]. For Aristotle, there is no universal answer to the question of how to interpret and apply equal treatment, but rather, the activity of debating that question is an essential component of living together as fellow citizens. These arguments can be fierce, "for battles and accusations arise when equals are assigned and possess unequal shares or when those who are not equal are assigned and possess equal shares" [26]. More recently, philosophers have explored what kinds of inequalities justify differential treatment in modern, democratic societies, and what kinds of justification can be offered for the preferential treatment of disadvantaged groups $[12,63,74,85]$, applying these questions to debates about compensation, victimization, meritocracy, and affirmative action [11, 18, 81, 97].

Contests over the meaning of equal treatment have also preoccupied legal theorists, especially in the United States where, as Jill Lepore [62] and Danielle Allen [9] argue, constitutional debates about qualifications for citizenship and voting stretch back to the Declaration of Independence. Because law is the domain in which competing principles are contested and debated, we use law to illustrate the stakes of normative debates about the meaning of equal treatment. Legal theorists have argued that "the mere idea of likeness or unlikeness" can be unhelpful, since "any two items in the world" can "be deemed alike in some respects and unalike in others", noting how equal treatment has often been used as a justification for exclusion and oppression [66, 67, 82]. Given this, many US constitutional lawyers have forcefully argued that constitutional law should reject the fictitious principle of neutrality, and that equal protection requires careful consideration of historic disadvantages and inequalities $[60,72,90]$.

Many legal scholars have applied debates about equal treatment to the concept and law of non-discrimination. Some have argued that the idea of discrimination does not depend on any concept of equality [47], others that it depends on a substantive concept of equality focused on power [34,36], and others still, in line with Aristotle and our own contribution, that contests over the meaning of equal treatment underpin contemporary debates about what makes discrimination wrong $[30,43,45]$ and how legal prohibitions against discrimination should be understood and enforced [16, 24, $54,86]$.

Debates about equal treatment and non-discrimination have recently been applied to data, algorithms, and machine learning. Early contributions have explored how normative concepts should apply to socio-technical systems [84], how discrimination law should apply to machine learning [17], and how the widespread use of machine learning may invite debates about the underlying purposes of discrimination law [57]. There have also been illuminating efforts to evaluate the legality of mathematical fairness definitions under current doctrines in US discrimination law [99]. Scholars have articulated illuminating descriptions of impossibility results $[23,59]$ and the principles that underpin group- and individual-level fairness definitions [21]. Others have argued that the application of the idea of discrimination to machine learning sharpen several longstanding criticisms of non-discrimination law and policy [46].

A few scholars have directly explored the tensions between equal treatment and mitigating inequality of impact. Friedler et al. [37] argue that whether fairness metrics should use protected traits depends on a judgement about whether differences in distributions of non-protected characteristics stem from structural disadvantages or other factors. This work directly relates to ours, since the treatmentas-blindness approach, mistakenly in our view, either explicitly or implicitly assumes uneven distributions of non-protected characteristics stem from luck or talent, rather than structural inequality.

Lipton et al. [64] examine machine learning methods that use protected categories during the training process, impose some chosen technical fairness constraint (such as demographic parity or equal opportunity), then use the trained model on data without access to protected categories. They call such approaches Disparate Learning Processes (DLPs), and note that DLPs often lead to unintended consequences. They consider an example in which men and women apply to a computer science course, and argue that if an algorithm is trained to make admissions decisions, constrained so as to accept more women than it otherwise would take, it will tend to modify its decision boundary to admit extra women who are 'male-like' in their other characteristics. This may prevent some women from admission who would have been admitted under the unconstrained algorithm. The authors note that the best solution may be explicitly to treat different groups differently.

This theme has been raised in other work. Dwork et al. [29] show that using a different classifier for each group - a clear violation of treatment-as-blindness - can yield significant benefits for disadvantage groups. Similarly, Zafar et al. [102] consider the advantages of using different classifiers and thresholds for different groups, providing a motivation based on the idea of envy-free division: they require that each group in aggregate prefers their classifier to switching to the other group's classifier. Ustun et al. [95] extend this work and provide formal fairness guarantees. In the context of hiring, Raghavan et al. [78] point to the "longstanding tension between disparate treatment and disparate impact: some techniques to control outcome disparities require the use of protected attributes, which may be considered disparate treatment," noting that the debate about if and when various forms of affirmative action may be justified in the design or use of machine learning models is as yet unsettled $[19,44,61]$. It is in part to that debate that we hope to contribute.

\section{THE PROBLEM \\ The Nature of the Problem}

Two principles generally inform approaches to non-discrimination: striving for equality of treatment and advancing various notions of equality of outcome. Different legal jurisdictions interpret and relate these principles in different ways. In this paper, we use the UK's Equality Act (2010) to explore the stakes of debates about the meaning of equal treatment and the tensions between the treatmentas-blindness interpretation and advancing outcome equality. The Equality Act prohibits two forms of discrimination. Lady Hale, former President of the UK Supreme Court, describes the relationship 
between these two forms of discrimination and the principles of equal treatment and equal outcomes:

"[T] he prohibition of direct discrimination aims to achieve equality of treatment. Indirect discrimination assumes equality of treatment - the PCP [decision-making system] is applied indiscriminately to all - but aims to achieve a level playing field, where people sharing a particular protected characteristic are not subjected to requirements which many of them cannot meet but which cannot be shown to be justified. The prohibition of indirect discrimination thus aims to achieve equality of results in the absence of such justification. It is dealing with hidden barriers which are not easy to anticipate or to spot" [42].

We apply UK law to a hypothetical discrimination suit against Facebook [5, 8, 48, 55]. Imagine the UK's equality regulator, the Equality and Human Rights Commission (EHRC), accuses a particular machine learning model called p(click) of being directly and indirectly discriminatory. Following the logic of this case will situate the tensions between direct and indirect discrimination within longstanding conflicts about how to interpret and relate equal treatment to advancing equality of outcomes [64, 78, 86, 101].

$\mathrm{P}$ (click) predicts the probability someone will click on a particular advertisement. $\mathrm{P}$ (click) uses data about which users click on which kinds of ads to learn which patterns of user behaviour and ad features accurately predict click probability, and then displays whichever ad someone is most likely to click on. Suppose there are gendered patterns in the kinds of job ads men and women tend to click on, such that women are more likely to click on ads for shorter-term, service sector and administrative jobs, whilst men are more likely to click on ads for longer-term, blue collar jobs. If the average income attached to the job ads men tend to click on is higher than the average income attached to the job ads women tend to click on, p(click) will consistently show job ads with higher average incomes to men than women.

The EHRC argues that p(click) directly discriminates, because it includes gender as an input variable. Direct discrimination prohibits decisions from being made because of protected characteristics, on the principle that protected characteristics are morally irrelevant to the distribution of benefits and burdens. Because p(click) "uses" a protected characteristic, gender, to treat people differently, show them different ads, it violates this principle. Facebook agreed to remove gender as an input but decided to conduct a statistical analysis of the training data. They found that users' gender could accurately be predicted from the thousands of other variables in the data. The EHRC argued that this analysis demonstrated p(click) was still directly discriminating. Information about gender was encoded in the training data, and as such, gender was still being used to make determinations, violating direct discrimination. Decision-making procedures which bury information about morally irrelevant protected characteristics, they argued, should not be immune from findings of direct discrimination.

Facebook issued a robust response. They argued that wellcalibrated machine learning models trained on representative datasets always reproduce statistical patterns in the training data, and those patterns often correlate with membership of social groups. $\mathrm{P}$ (click) shows job ads with lower average incomes to women than men because gender correlates with the types of job ads people tend to click on, and the types of jobs women disproportionately click on tend to have lower average incomes. The reason almost every variable in their training data correlates with gender is that gender conditions the opportunities people are afforded. Forcing machine learning models into a false form of blindness by deliberately hiding the complex correlations between protected characteristics and other variables, Facebook argued, would not change underlying social inequalities. Precisely because it is a well-calibrated model that treats people equally with respect to the task of predicting click probability, $\mathrm{p}$ (click) replicates persistent inequalities among genders.

The EHRC decided not to press the direct discrimination case. Facebook's personalised advertising system did not subject all women to the relative disadvantage of being shown lower-paid job adverts than men, the problem was simply that the ads shown to women were for jobs that were on average lower paid than those shown to men. This meant p(click) failed the "exact correspondence" test used in UK law to detect proxies for protected characteristics in direct discrimination, and so, the charge of direct discrimination would be unlikely to stand. ${ }^{1}$

Instead the EHRC argued p(click) was indirectly discriminating. They felt persistent disparities in the average income of the job ads shown to men and women were persuasive evidence of prima facie indirect discrimination and required Facebook to justify those disparities. While the ERHC accepted that using click predictions to deliver ads which are relevant and useful was a legitimate aim, they argued the disparities produced by p(click) could only be justified as a proportional means of achieving that aim if Facebook could demonstrate there was no reasonable alternative machine learning system which would achieve that aim with less or no disparate impact. Facebook agreed to explore reasonable alternatives and submit a report summarizing their findings.

Facebook identified two possible courses of action that might reduce outcome disparities $[25,27]$. The first was to impose a version of demographic parity. Facebook could require the average income of job ads shown to women to be not more than 5 percent lower than the average income of job ads shown to men. While setting a maximum disparity would achieve a clear reduction in the disparities between the average income of job ads shown to men and women, it would pose two problems. First, unless underlying patterns in click behaviour changed, imposing demographic parity would considerably reduce $\mathrm{p}$ (click)'s accuracy [23, 25, 59]. This would not only reduce revenue from advertisers, it would lead to more people seeing ads for jobs they were not interested in and

\footnotetext{
${ }^{1}$ Further exploration is required of the idea that models like $\mathrm{p}$ (click) might constitute "proxy cases" of direct discrimination, because p(click) subjects women to the particular disadvantage of the "greater risk" of being shown a job advert with a lower average income than those shown to men. Consider Lady Hale's judgement in Coll: "... the question of comparing like with like must always be treated with great care - men and women are different from one another in many ways, but that does not mean that the relevant circumstances cannot be the same for the purpose of deciding whether one has been treated less favourably than the other. Usually, those circumstances will be something other than the personal characteristics of the men and women concerned, something extrinsic rather than intrinsic to them." Lady Hale, R (on the application of Coll) v Secretary of State for Justice (UKSC May 24, 2017). Because Facebook's advertising system is personalised, not all women suffer the relative disadvantage of the "greater risk" of being shown lower-paid job adverts. The disadvantage centres on averages and is not an exact correspondence. It is likely that women who tend to click on higher-income job adverts may be shown job adverts with a higher average income than those shown to men. Personalized machine learning models will rarely meet "exact correspondence" tests $[3,68]$.
} 
more employers missing the opportunity to receive applications from qualified and interested candidates [25, 28, 65, 77].

More fundamentally, imposing demographic parity might itself amount to direct discrimination. Imposing a maximum disparity across gender would treat people differently, deliberately changing which job ads they see, on the basis of gender, which would involve treating people differently on the basis of a morally irrelevant characteristic, violating the treatment-as-blindness interpretation of equal treatment.

The second option was to put gender back in as an input variable. Since $\mathrm{p}$ (click) was producing persistent disparities in the average income of job ads across gender, it was showing that gender is statistically relevant to predicting click probability. Including gender would enable the model to make more fine-grained predictions in full knowledge of underlying disparities, rather than being blinded to them. As Facebook expected, experiments showed that including gender as a feature narrowed the disparities between the average income of job ads shown to men and women, although it did not eliminate them [58].

The problem was the EHRC had already argued this would constitute direct discrimination, even though Facebook had argued that the disparities $\mathrm{p}$ (click) produced were the result of underlying patterns of gender inequality. Facebook faced a choice: either exclude gender, but accept higher disparities in the average income of job ads shown to men and women; or include gender and narrow those disparities, but accept the risk of violating direct discrimination $[29,78,99,102]$.

Cases like p(click) make visible the tension between the treatment-as-blindness interpretation of equal treatment and advancing equality of outcomes. When $\mathrm{p}$ (click) predicts that the kinds of job ads men tend to click on have higher average incomes than those women tend to click, $\mathrm{p}$ (click) is simply replicating patterns in the underlying training data that reflect enduring and pervasive gender pay gaps $[39,40]$. If $\mathrm{p}$ (click) is well-calibrated across gender and other social groups, trained on a representative and expressive dataset, $\mathrm{p}$ (click) will show job ads with higher average incomes to men than women. Men will then click on job ads with higher average incomes, producing more training data that reflects gender disparities, which $\mathrm{p}$ (click) will reflect in its predictions, and so on $[8,80,94]$.

$\mathrm{P}$ (click) unearths these patterns precisely because it treats men and women as equals, differentiating among them only to the extent they are statistically different in terms of the prediction task. In an automated system like Facebook's advertising system, requiring $\mathrm{p}$ (click) to respect equal treatment not only replicates but reinforces inequalities between men and women. In an unequal world, statistical models that respect equal treatment-as-blindness will differentially impact those who are unequal, and over time, may compound enduring patterns of social inequality. When equal treatment is interpreted to require blindness, imposing equal treatment in machine learning may entrench inequalities of outcome.

\section{The Scope of the Problem}

$\mathrm{P}($ click $)$ is an extremely common kind of case in machine learning. The outcome $\mathrm{p}$ (click) is trained to predict is distributed unequally across social groups: click probability for high- and low-income job ads is unevenly distributed across gender. This is not an inexorable fact of nature, nor is it necessarily the result of statistical bias in the training data, it is a social fact, the result of enduring patterns of inequality across gender $[39,40]$. When a statistical model predicts an outcome that is unevenly distributed across groups, if the model is well-calibrated and trained on a representative dataset, its predictions will reflect that uneven distribution, and the use of those predictions in decision-making can entrench the underlying patterns. Precisely because they treat people equally with respect to a prediction task, the predictions of statistical models often reflect uncomfortable social inequalities, in the case of $\mathrm{p}$ (click), enduring social inequalities between men and women.

In an unequal world, imposing equal treatment-as-blindness may guarantee inequality of impact, and conversely, addressing unequal impact may require awareness and sensitivity to difference that violates treatment-as-blindness. This tension is not unique to machine learning - it is the general problem that treating as equals those who are not in fact equals may reproduce and compound the inequalities between them - but machine learning makes the problem visible and tractable. The problem statement, then, is as follows.

(1) If treatment-as-blindness: If equal treatment is interpreted to require some form of blindness, prohibiting decisions from being made "because of" protected characteristics; (2) And social inequality: And an outcome is unevenly distributed across groups who share a protected characteristic; (3) Then a well-calibrated machine learning model trained on a representative dataset which predicts that outcome can: i. Either entrench social inequality: Respect treatment-as-blindness but replicate the uneven distribution of the outcome in its predictions, which, when they are used to make decisions, will disproportionately impact disadvantaged groups. ii. Or violate treatment-as-blindness: Use awareness of difference to address the underlying inequalities, often by considering the protected characteristic, but violate treatment-as-blindness.

An unbiased and accurate statistical model will reflect whatever patterns of social inequality exist in the data on which it was trained. If those patterns tend to correlate with protected characteristics like race and gender, then the use of that model's predictions in decision-making will have disparate impact across protected groups precisely because it treats people equally with respect to that prediction task. The model has learned that our society is ridden with systemic inequalities that shape the distribution of the outcome it has been asked to predict. Disadvantaged groups are, in a sense, disproportionately burdened by the use of patterns in data to make decisions - by statistics - sharpening exactly the questions Aristotle asked: What kinds of differences justify differential treatment? And differential treatment of what kinds? As the use of predictions in decision-making becomes increasingly common, ancient debates about how the meaning of equal treatment may become increasingly urgent [10, 34, 36, 54].

\section{SOLUTIONS}

We now consider how to respond to this problem. We illustrate the normative stakes of resolving it by first considering technical solutions, then exploring three policy options. All consider cases in which a machine learning model ( $\mathrm{p}(\mathrm{click}))$ predicts an outcome 
(click probability) that is unevenly distributed across social groups (gender) and is used to make decisions (distribute job adverts) that can compound existing disadvantage (exacerbate unequal employment opportunities across gender).

\section{Technical}

Technical solutions would involve methods for training and deploying machine learning models that reduce persistent inequalities of outcome without violating treatment-as-blindness.

One common approach is Disparate-Learning Processes (DLPs). These approaches use protected categories during training, impose some fairness constraint (such as demographic parity or equal opportunity), then use the trained model on data without access to protected categories. Using protected characteristics during training but not to generate predictions would be like Facebook including gender as a variable in p(click)'s training process but excluding gender from the data about particular users that is used to generate click predictions. DLPs use protected characteristics in training process in different ways, such as to narrow the set of acceptable prediction rules, pre-process the training data, or constrain the predictor $[50,76,103]$.

There are at least three problems with DLPs. First, DLPs may not in fact satisfy the treatment-as-blindness requirement. Whether a model trained on gender but that does not use gender in its predictions should be immune from the charge of direct discrimination or disparate treatment is an open question [64]. More generally, the effort to remove sensitive variables in machine learning is often ineffective and can have counter-productive consequences. In the case of $\mathrm{p}$ (click), if gender correlates with many of the variables social media companies have available, attempting to make $\mathrm{p}$ (click) blind to gender may sacrifice accuracy and may also not benefit women in the long run [65].

Second, and more fundamentally, as Lipton et al. [64] show, the optimal decision rule in terms of both accuracy and benefits to the disadvantaged group often requires awareness and sensitivity to difference, or in legal terms, direct discrimination and disparate treatment. In the case they examine, the optimal decision rule involves selecting group-specific thresholds and constraining the selection of those thresholds to benefit the disadvantaged group. Building machine learning models to maximally benefit disadvantaged groups, therefore, may require violating notions of treatment-as-blindness $[29,95,102]$.

Third and finally, DLPs illustrate the importance of separating prediction from decision-making. The fact that a predictor uses a protected trait should not be understood to entail the conclusion that a decision-making system which uses that predictor violates disparate treatment. Conversely, the most effective way to ensure a decision-making system mitigates disparate impact may not be to constrain the predictor used to make decisions. In machine learning, sacrificing subgroup calibration or accuracy may often harm the disadvantaged as well as the advantaged group, in our case, resulting in more women being shown job ads in which they are not in fact interested. As Kleinberg et al. [56] put it: "a preference for fairness should not change the choice of estimator". It may often be unwise to apply intuitions about the desirable goals of a decision-making system to the design of machine learning models.
Another valuable approach is to develop proxies that better reflect the underlying goals of a decision-making system. Consider $\mathrm{p}$ (click) again. To reduce inequalities of outcome, Facebook could invest in developing more sophisticated proxies for measuring the value to users of seeing a job ad. They could ask employers to complete satisfaction surveys about employees they hire and employees to complete satisfaction surveys about jobs they apply to and take up. Facebook could then analyse data from these surveys to produce a combined score for employer and employee satisfaction. Facebook could combine this score with other proxies that capture components of job quality: how far the advertised job was from someone's home, the income of that job relative to someone's current or previous income, the average ratings of the prospective employer by past employees, and so on. The satisfaction scores could be combined with job quality scores and included within the objective function that Facebook's advertising system would maximize:

ad score $=p(\text { click })^{*}(p($ job quality score $)+p($ employee satisfaction score))

Developing better proxies is an important strategy for reducing outcome disparities without violating equal treatment. But there are fundamental limits to how well we can measure what we care about: there is no way to measure talent that separates out all the factors influenced by structural inequalities, including those associated with protected characteristics like race or gender. While developing better proxies should be encouraged, doing so will not address the fundamental tension between treatment-as-blindness and advancing equality of outcomes. No technical approach can address the philosophical question and policy choice about when, how, and why equal treatment should be interpreted and applied.

\section{Policy}

Thinking through the policy alternatives before us can help illuminate the normative stakes of how we interpret and apply the principle of equal treatment in the governance of decision-making. We explore three high-level policy approaches that respond to the tension between the treatment-as-blindness interpretation of equal treatment and advancing equality of outcome: status quo, industryled, and government-led approaches.

Option 1: Status Quo. The status quo approach continues to insist the regulation of decision-making be motivated by some form of equal treatment-as-blindness and prohibit the use of protected characteristics in decision-making.

In countries like the US and UK where the imaginative grip of formalistic interpretations of equal treatment is strong, fear of violating prohibitions against direct discrimination or disparate treatment may prevent organizations from exploring different ways of designing and using statistical systems to reduce persistent inequalities of outcome.

For instance, consider a real-world discrimination suit launched against Facebook by the US Department of Housing and Urban Development (HUD). HUD has accused Facebook of discrimination on the grounds that ads were delivered to users "because of" their protected characteristics [48]. It is unclear what HUD intends this to mean. The obvious interpretation is simply that HUD believes 
Facebook has used protected characteristics or close proxies to distribute ads. If so, given HUD's recent proposed rule [49], which effectively immunizes organizations from discrimination charges if they remove protected traits and close proxies from their systems, Facebook would appear to be able to address the charge by removing protected traits and close proxies from their advertising delivery and targeting systems [75, 83]. If HUD's motivating concern is the unequal impact of Facebook's advertising system, addressing the concern may require precisely the opposite course of action, that is, deliberately using protected characteristics to narrow impact disparities, potentially delivering ads to people "because of" protected characteristics and violating the original charge HUD levelled at Facebook.

Even in the UK, where interpretations of equal treatment are often assumed to be more sensitive to disadvantage, the absence of a "general justification defence for direct discrimination on grounds of race or sex... precludes the possibility of arguing that the purpose was the benevolent one of redressing disadvantage," leading "to a remarkably restrictive approach to any provision which exhibits an explicit preference on grounds of race or sex" $[35,69]$. The result is that US civil rights law and UK equality law may at present effectively prevent Facebook from pursuing many interventions to reduce persistent inequalities produced by its advertising delivery system that disproportionately harm disadvantaged groups [8]. It may become all too easy for companies with unilateral market power to justify disparities produced by machine learning models by demonstrating that alternative ways of designing those models to reduce outcome disparities would themselves constitute disparate treatment or direct discrimination.

Our point here is not to offer original interpretations of US or UK law, but to emphasise that insofar as laws that govern decisionmaking are confined by the treatment-as-blindness interpretation of equal treatment, the choice to settle for the status quo is not normatively neutral. As we have argued, when an outcome is unevenly distributed across advantaged and disadvantaged groups, imposing equal treatment-as-blindness on machine learning models which predict that outcome will guarantee the model replicates the uneven distribution of the outcome. Without intervention or oversight, using those predictions to make decisions may disproportionately harm disadvantaged groups. What's more, these unequal impacts may be extremely difficult to discern and interrogate, since they will be hidden behind the veil of neutrality statistics is often taken to confer. If policy makers wish for companies like Facebook to exert their energy not on justifying outcome disparities, but on working out how to address them, reforms to the current policy approach will be required. Imposing treatment-as-blindness in the context of persistent social inequalities will often ensure decisionmaking systems entrench objectionable inequalities of outcome $[13,35,98,100]$.

We suggest that the underlying purpose of equality, antidiscrimination, and civil rights laws is to ensure relationships of power between citizens do not become ossified and to promote mutual flourishing. These laws shift the burdens of inequality from the shoulders of disadvantaged groups onto organizations with the resources to address them, empowering citizens to be co-equals and co-creators, rather than subject to unchosen structures of domination and oppression. The treatment-as-blindness view risks making equality and civil rights laws into constraints that prevent organisations from using protected characteristics to confront structures of domination and oppression. The danger is these laws become tools that entrench inequality, rather than tools that empower and incentivize organisations to explore how to use machine learning to address inequality.

Option 2: Industry-led. The second approach would remove constraints on organizations committed to exploring how best to use machine learning to advance equality of outcomes. It would allow organisations to make their own contextual judgements about how to interpret and implement equal treatment in their decisionmaking while imposing more robust mechanisms to structure accountability, auditability, and transparency. This approach would not impose any specific duties to reduce persistent inequalities of outcome but would provide organisations with the freedom to explore how best to do so.

Removing treatment-as-blindness constraints would require a shift in how equal treatment is interpreted and applied, away from treatment-as-blindness and towards treatment-as-awareness. We believe there are strong normative and practical reasons for such a shift.

The principle of equal treatment denotes a formal relationship that lacks substantive content. As Aristotle argued, living together as equal citizens requires us to debate who should be considered similar to whom and what kinds of differences justify differential treatment $[9,38,74]$. As one legislator in the UK explains, the principle of "treating like cases alike and unlike cases differently is a general axiom of rational behaviour," but "the very banality of the principle must suggest a doubt as to whether merely to state it can provide an answer" to judgements in particular cases.

"Of course persons should be uniformly treated, unless there is some valid reason to treat them differently. But what counts as a valid reason for treating them differently? And, perhaps more important, who is to decide whether the reason is valid or not? Must it always be the courts? The reasons for not treating people uniformly often involve, as they do in this case, questions of social policy on which views may differ. These are questions which the elected representatives of the people have some claim to decide for themselves" [1]. ${ }^{2}$

In countries like the US and UK, a redefinition of the meaning of equal treatment may be long overdue. Several conservative justices in the US have suggested that the struggle over how to interpret equal treatment will be high on their agenda in the coming years. As Supreme Court Justice Anthony Scalia wrote: "the war between disparate impact and equal protection will be waged sooner or

\footnotetext{
${ }^{2}$ Lord Hoffman, delivering the Privy Council's advice in Matadeen v Pointu [1]. He went on to argue that it should generally be the legislature not courts that articulates broad judgements about who is similar to whom in different contexts. This too may become an increasingly important question. "The fact that equality of treatment is a general principle of rational behaviour does not entail that it should necessarily be a justiciable principle - that it should always be the judges who have the last word on whether the principle has been observed. In this, as in other areas of constitutional law, sonorous judicial statements of uncontroversial principle often conceal the real problem, which is to mark out the boundary between the powers of the judiciary, the legislature and the executive in deciding how that principle is to be applied... A self-confident democracy may feel that it can give the last word, even in respect of the most fundamental rights, to the popularly elected organs of its constitution. The United Kingdom has traditionally done so; perhaps not always to universal satisfaction, but certainly without forfeiting its title to be a democracy."
} 
later... it behooves us to begin thinking about how - and on what terms - to make peace between them" [2], a struggle whose stakes are considerably raised by the widespread use of machine learning.

The treatment-as-blindness view, as Sandra Fredman argues, fails to "address the historical disadvantage suffered by those subject to discrimination" [41]. While treatment-as-blindness has "traditionally been" defended "and legitimated on the grounds that [it] further[s] the liberal goals of state neutrality, individualism, and the promotion of autonomy... formal equality before the law," such "neutrality [can] reinforce dominant values or existing distributions of power" [34]. The structures of domination and subordination to which women and people of color have historically been subject constitutes exactly the kind of difference that justifies the differential treatment of advantaged and disadvantaged groups, as Owen Fiss, Justice Henry Blackmun, Justice Sonya Sotomayer, and the Black Lives Matter movement, among many others, have argued.

Machine learning sharpens this critique. In our running example, $\mathrm{p}$ (click) is trained on data which reflects gender disparities: women tend to click on ads for shorter-term, service sector and administrative jobs, and the average income attached to those jobs is lower than the average income attached to ads for jobs men tend to click on. $\mathrm{P}$ (click) reflects those social inequalities in its predictions, showing women adverts for jobs with lower average incomes than men There is nothing intrinsically wrong with $\mathrm{p}$ (click) using gender as an input in the training process or the model itself: gender is a category of disadvantage, and as such, men and women are different in precisely the sense that justifies their differential treatment.

The treatment-as-awareness view holds that the principle of equal treatment requires moral awareness of, and empirical sensitivity to, differences that justify differential treatment. In the case of $\mathrm{p}$ (click), if including gender as an input in the model narrows disparities between men and women, gender not only can but should be included. Addressing pervasive social inequalities requires deliberately taking account categories of disadvantage when making decisions about the distribution of benefits and burdens. If machine learning helps to broaden "recognition of those inequalities, and of the tendency for equal treatment of the unequally situated to exacerbate, rather than challenge, inequality" this could "do much to diffuse the unease which characterises... discussions of "positive discrimination"” [69].

The treatment-as-awareness view provides a framework for justifying differential treatment, by reasserting Aristotle's dictum that unlike cases should be treated "in proportion to their unlikeness" $[10,26,82]$. The requirement to treat people differently in proportion to their differences focuses our attention on empirical investigations and moral evaluations of the dimensions of difference $[36,66]$. It also adds clarity about the directionality of justifications of differential treatment. The fact that race is a category of persistent and relevant disadvantage justifies positive action on behalf of those who are disadvantaged, African Americans, not those who are advantaged, white Americans. The fact that gender is a category of disadvantage justifies positive action not on the grounds of gender, but on behalf of women, because it is women who are subject to the myriad consequences of that disadvantage. Treatment-as-awareness rejects the moral equivalence of decisionmaking systems that cause disparate impact to advantaged and disadvantaged groups [2].
While this option would enable organisations to apply contextsensitive judgements about equal treatment, it should also be accompanied by more robust structures for securing accountability, auditability, and transparency. Organizations should be required to explain to citizens and regulators in concrete terms how they are interpreting and implementing equal treatment in their decisionmaking. This could extend to sandboxing structures that would permit industry to innovate and experiment with methods of building and using machine learning models that advance racial justice and gender equality, while enabling regulators to set defined parameters, observe the process of experimentation, and interrogate the results. Lessons learned from this experimentation could then inform policy changes at a wider level [15, 93].

Option 3: Government-led. Like the previous approach, the third option would also define equal treatment in terms of awareness rather than blindness to disadvantage. However, instead of simply removing constraints that prevent organisations from using protected characteristics, the third approach would impose positive duties that require organisations to proactively consider how best to advance equality of outcome across protected groups as they design and deploy machine learning systems. This approach would clarify when positive duties should be prioritized over prohibitions against violating treatment-as-blindness [54].

This approach could build on existing positive duties, like affirmative action in the US or the Public Sector Equality Duty (PSED) in the UK $[4,6,51,53,73,88,92]$. At present, positive duties generally apply only to state bodies in the exercise of their public functions. For example, the PSED requires public bodies to give due regard to the need to eliminate unlawful discrimination, harassment and victimisation and other prohibited conduct; advance equality of opportunity; and foster good relations between people who share a protected characteristic and those who do not. ${ }^{3}$ The duty has in practice come to require public bodies to carry out an impact assessment of the differential effects of proposed policy measures before they are implemented.

Building positive duties into the regulation of decision-making may be critical to ensure the widespread use of data-driven technologies advances equality, rather than entrenches inequality. Deliberate and early-stage consideration of how to advance equality is often required when building predictive tools and integrating them into decision-making systems. As Virginia Eubanks argues, machine learning "tools... left on their own, will produce towering inequalities unless" they are "built to explicitly dismantle structural inequalities, their increased speed and vast scale [will] intensify them dramatically" [31]. Given this, positive duties may be "the most appropriate way to advance equality and to fight discrimination, including indirect discrimination" [92].

The government-led approach would define a set of decision contexts in which organizations would have positive duties to consider how best to advance equality of outcome across protected and non-protected groups, and to advance equality of opportunity. We describe this as a Positive Equality Duty (PED), which would play

\footnotetext{
${ }^{3}$ The EHRC describe these as the three arms of the PSED: Removing or minimising disadvantages suffered by people due to their protected characteristics; taking steps to meet the needs of people from protected groups where these are different from the needs of other people; encouraging people from protected groups to participate in public life or in other activities where their participation is disproportionately low.
} 
a distinctive role in the logic of equality and civil rights law. The PED would serve as a justification for the differential treatment of those who share a protected characteristic and those who do not, in narrowly defined contexts and subject to clear accountability requirements. In contexts where the PED applies, organizations would be able to take actions to reduce persistent inequalities of outcome without fear of violating direct discrimination, provided they could demonstrate those actions constituted reasonable measures to discharge their PED. PEDs would, in other words, give organisations a targeted license to violate equality of treatment, including by using protected characteristics, in order to advance equality of outcome.

The PED should extend beyond state bodies and apply to all decision contexts that have profound impacts on citizens' lives. Positive duties are designed to ameliorate and address disadvantage, and so, those duties should apply to areas of decision-making and organisations with the power and resources to address that disadvantage, regardless of whether they are state or private bodies. This may be especially important for monopolistic technology platforms whose power matches those of many government bodies, when it can be extremely difficult for regulators or claimants to demonstrate those companies have violated discrimination laws in how they have designed and deployed machine learning systems [70, 71, 99] The legislature should specify the exact verticals in which the duty applies, but at minimum, it should include private organisations engaged in employment, housing, healthcare, access to credit and other financial instruments, and the control and design of public spaces. When and how positive duties should be imposed in these areas would require consensus building and collaboration among government, civil society, regulators and industry. Targeted positive duties should also be time-limited by clear, public measures of outcome disparities that organizations would be required to report on a regular basis.

The effects of PEDs would be significant. They would redirect organisations' energy from working out how to justify outcome disparities, towards working out how to address them. Facebook, for instance, would not be able to objectively justify $\mathrm{p}$ (click) by showing that reasonable alternatives that reduce outcome disparities would themselves violate direct discrimination, since the positive duty would establish that reasonable efforts to advance equality constitute a prima facie justification of direct discrimination. Instead, Facebook would be required to explore the full set of alternative ways of designing their advertising system using statistical systems like p(click) so as to reduce outcome disparities, whilst also meeting their fundamental business objectives, including by using protected characteristics.

This would change the incentives of the existing policy regime for regulating decision-making, away from encouraging organizations to justify machine learning systems that entrench disadvantage and towards exploring how they can most effectively use machine learning to address disadvantage. Ensuring that organizations interrogate whether what appears to be direct discrimination or disparate treatment addresses or compounds disadvantage may be essential for the advancement of racial justice, gender equality, and socio-economic opportunity as the use of machine learning models in decision-making becomes increasingly common.

\section{DISCUSSION}

While there are benefits and drawbacks to both the industry-led and government-led approach, we remain agnostic about which should be pursued, noting that different variations of these options may suit different jurisdictions. Our stylized alternatives are designed to illustrate the spectrum of alternatives for policy makers, the underlying normative principles that motivate those alternatives, and their distinct implications for how machine learning models are built and deployed in decision-making.

Our central argument is that interpretations of equal treatment wedded to the treatment-as-blindness view will not only fail to promote equality of outcomes, it may actively prevent organizations from pursuing many approaches that would leverage the power of machine learning to advance equality of outcome among protected groups. We believe it is vital that policy makers appreciate this implication of the status quo and engage with the pressing underlying philosophical problem.

Each stage of this argument raises numerous avenues for further research and investigation. While our contribution is primarily to connect normative political philosophy with recent debates in law and computer science, moral and political debates about how to interpret and apply equal treatment are far from settled, especially in the context of machine learning. In the domain of law and policy, for instance, the industry-led or government-led approach would require wholesale updates to equality and civil rights law, as well as rigorous consideration of who should decide, how they should decide, and in what contexts it should be permissible to use protected characteristics to reduce inequalities of outcome.

While the PED proposal would encourage a general shift in equality and civil rights law away from ex post enforcement by courts focused on individual remedies, towards ex ante enforcement by regulators focused on structural remedies, it invites further consideration of the mechanisms by which regulators would in practice enforce this duty. This should extend to a critical evaluation of the possibilities and limits of impact assessments as mechanisms for interrogating how an organisation interprets and applies equal treatment in machine learning $[14,20,96]$.

\section{ACKNOWLEDGEMENTS}

As a visiting researcher in Facebook's Responsible AI team, Josh Simons is a paid consultant to Facebook. The views reflected here are the authors' alone, and Facebook had no role in the production or review of this report.

Adrian Weller acknowledges support from a Turing AI Fellowship under grant EP/V025379/1, The Alan Turing Institute under EPSRC grant EP/N510129/1 and TU/B/000074, and the Leverhulme Trust via the CFI.

\section{REFERENCES}

[1] 1999. Matadeen v. Pointu.

[2] 2009. Ricci v. DeStefano.

[3] 2017. R (on the application of Coll) v. Secretary of State for Justice.

[4] Morris B Abram. 1986. Affirmative Action: Fair Shakers and Social Engineers. Harvard law review 99, 6 (1986), 1312-1326.

[5] Ifeoma Ajunwa. 2019. Automated Employment Discrimination 34 HARV. JL \& TECH. _ (forthcoming 2021). FL \& TECH. _ (forthcoming 2021).(March 15, 2019) 34 (2019).

[6] Ifeoma Ajunwa. 2019. The paradox of automation as anti-bias intervention. Cardozo L. Rev. 41 (2019), 1671. 
[7] Sven Patric Aldeberg, Fiona Jane Liebe Saunders Watson, Malcolm Clive North, et al. 1999. National Coalition for Gay and Lesbian Equality V. Minister of Home Affairs*[December 2, 1999]+ Cite as 39 ILM 798 (2000). (1999).

[8] Muhammad Ali, Piotr Sapiezynski, Miranda Bogen, Aleksandra Korolova, Alan Mislove, and Aaron Rieke. 2019. Discrimination through Optimization: How Facebook's Ad Delivery Can Lead to Biased Outcomes. Proceedings of the ACM on Human-Computer Interaction 3, CSCW (2019), 1-30.

[9] Danielle Allen. 2014. Our declaration: A reading of the Declaration of Independence in defense of equality. WW Norton \& Company.

[10] Danielle S Allen. 2003. The world of Prometheus: The politics of punishing in democratic Athens. Princeton University Press.

[11] Danielle S Allen. 2006. Talking to strangers. anxieties of citizenship since brown v board of education. Number 23. Editions Hermann.

[12] Elizabeth Anderson. 2013. The imperative of integration. Princeton University Press.

[13] Elizabeth S Anderson. 1999. What is the Point of Equality? Ethics 109, 2 (1999), 287-337.

[14] Equality Impact Assessment. 2012. Equality Impact Assessment. Dementia 1 (2012), 2-135.

[15] Financial Conduct Authority. 2015. Regulatory sandbox. online $<$ https://www. fca. org. uk/publication/research/regulatory-sandbox. pdf $>$ (last accessed 1 October 2016) (2015).

[16] Jack M Balkin and Reva B Siegel. 2003. The American civil rights tradition Anticlassification or antisubordination. Issues in Legal Scholarship 2, 1 (2003).

[17] Solon Barocas and Andrew D Selbst. 2016. Big data's disparate impact. Calif. L. Rev. 104 (2016), 671.

[18] Herman Belz. 1990. Equality transformed: A quarter-century of affirmative action. Vol. 15. Transaction Publishers.

[19] Jason R Bent. 2019. Is Algorithmic Affirmative Action Legal? Georgetown Law Journal, Forthcoming (2019).

[20] Reuben Binns. 2017. Data protection impact assessments: a meta-regulatory approach. International Data Privacy Law 7, 1 (2017), 22-35.

[21] Reuben Binns. 2020. On the apparent conflict between individual and group fairness. In Proceedings of the 2020 Conference on Fairness, Accountability, and Transparency. 514-524.

[22] Henry Blackmun. 1978. Regents of the University of California v. Bakke.

[23] Alexandra Chouldechova. 2017. Fair prediction with disparate impact: A study of bias in recidivism prediction instruments. Big data 5, 2 (2017), 153-163.

[24] Hugh Collins and Tarunabh Khaitan. 2018. Foundations of indirect discrimination law. Bloomsbury Publishing.

[25] Sam Corbett-Davies and Sharad Goel. 2018. The measure and mismeasure of fairness: A critical review of fair machine learning. arXiv preprint arXiv:1808.00023 (2018).

[26] Roger Crisp. 2014. Aristotle: Nicomachean Ethics. Cambridge University Press.

[27] William Dieterich, Christina Mendoza, and Tim Brennan. 2016. COMPAS risk scales: Demonstrating accuracy equity and predictive parity. Northpointe Inc (2016).

[28] Cynthia Dwork, Moritz Hardt, Toniann Pitassi, Omer Reingold, and Richard Zemel. 2012. Fairness through awareness. In Proceedings of the 3rd innovations in theoretical computer science conference. 214-226.

[29] Cynthia Dwork, Nicole Immorlica, Adam Tauman Kalai, and Max Leiserson. 2018. Decoupled Classifiers for Group-Fair and Efficient Machine Learning (Proceedings of Machine Learning Research, Vol. 81), Sorelle A. Friedler and Christo Wilson (Eds.). PMLR, 119-133.

[30] Benjamin Eidelson. 2015. Discrimination and disrespect. Oxford University Press.

[31] Virginia Eubanks. 2018. Automating inequality: How high-tech tools profile, police, and punish the poor. St. Martin's Press.

[32] Michael Feldman, Sorelle A Friedler, John Moeller, Carlos Scheidegger, and Suresh Venkatasubramanian. 2015. Certifying and removing disparate impact. In proceedings of the 21th ACM SIGKDD international conference on knowledge discovery and data mining. 259-268.

[33] Owen M Fiss. 1976. Groups and the equal protection clause. Philosophy \& Public Affairs (1976), 107-177.

[34] Sandra Fredman. 2001. Equality: a new generation? Industrial Law fournal 30, 2 (2001), 145-168.

[35] Sandra Fredman. 2014. Addressing disparate impact: Indirect discrimination and the public sector equality duty. Industrial Law fournal 43, 3 (2014), 349-363.

[36] Sandra Fredman. 2016. Substantive equality revisited. International fournal of Constitutional Law 14, 3 (2016), 712-738.

[37] Sorelle A Friedler, Carlos Scheidegger, and Suresh Venkatasubramanian. 2016. On the (im) possibility of fairness. arXiv preprint arXiv:1609.07236 (2016).

[38] Raymond Geuss, Quentin Skinner, et al. 1996. Aristotle: The Politics and the Constitution of Athens. Cambridge University Press.

[39] Claudia Goldin. 2014. A grand gender convergence: Its last chapter. American Economic Review 104, 4 (2014), 1091-1119.

[40] Claudia Goldin, Sari Pekkala Kerr, Claudia Olivetti, and Erling Barth. 2017. The expanding gender earnings gap: Evidence from the LEHD-2000 Census.
American Economic Review 107, 5 (2017), 110-14.

[41] Evadné Grant. 2007. Dignity and equality. (2007)

[42] Lady Hale. 2017. Essop v. Home Office. , 27 pages.

[43] Deborah Hellman. 2008. When is discrimination wrong? Harvard University Press.

[44] Deborah Hellman. 2020. Measuring algorithmic fairness. Va. L. Rev. 106 (2020), 811.

[45] Deborah Hellman and Sophia Moreau. 2013. Philosophical foundations of discrimination law. OUP Oxford.

[46] Anna Lauren Hoffmann. 2019. Where fairness fails: data, algorithms, and the limits of antidiscrimination discourse. Information, Communication \& Society 22, 7 (2019), 900-915.

[47] Elisa Holmes. 2005. Anti-Discrimination Rights Without Equality. The Modern Law Review 68, 2 (2005), 175-194.

[48] HUD. 2019. Charge Sheet. https://www.hud.gov/sites/dfiles/Main/documents/ HUD_v_Facebook.pdf.

[49] HUD. 2019. HUD's Implementation of the Fair Housing Act's Disparate Impact Standard. https://www.federalregister.gov/documents/2019/08/19/201917542/huds-implementation-of-the-fair-housing-acts-disparate-impactstandard\#h-9.

[50] Faisal Kamiran, Toon Calders, and Mykola Pechenizkiy. 2010. Discrimination aware decision tree learning. In 2010 IEEE International Conference on Data Mining. IEEE, 869-874

[51] Ira Katznelson. 2005. When affirmative action was white: An untold history of racial inequality in twentieth-century America. WW Norton \& Company.

[52] Ibram X Kendi. 2019. How to be an antiracist. One world.

[53] Randall Kennedy. 2013. For discrimination: Race, affirmative action, and the law. Pantheon.

[54] Tarunabh Khaitan. 2015. A theory of discrimination law. OUP Oxford.

[55] Pauline T Kim and Sharion Scott. 2018. Discrimination in online employment recruiting. . Louis ULF 63 (2018), 93.

[56] Jon Kleinberg, Jens Ludwig, Sendhil Mullainathan, and Ashesh Rambachan. 2018. Algorithmic fairness. In AEA papers and proceedings, Vol. 108. 22-27.

[57] Jon Kleinberg, Jens Ludwig, Sendhil Mullainathan, and Cass R Sunstein. 2018. Discrimination in the Age of Algorithms. Fournal of Legal Analysis 10 (2018).

[58] Jon Kleinberg and Sendhil Mullainathan. 2019. Simplicity creates inequity: implications for fairness, stereotypes, and interpretability. In Proceedings of the 2019 ACM Conference on Economics and Computation. 807-808.

[59] Jon Kleinberg, Sendhil Mullainathan, and Manish Raghavan. 2016. Inherent trade-offs in the fair determination of risk scores. arXiv preprint arXiv:1609.05807 (2016).

[60] Kathryn Kolbert and David H Gans. 1993. Responding to Planned Parenthood v. Casey: Establishing Neutrality Principles in State Constitutional Law. Temple L. Rev. 66 (1993), 1151.

[61] Joshua A Kroll, Solon Barocas, Edward W Felten, Joel R Reidenberg, David G Robinson, and Harlan Yu. 2016. Accountable algorithms. U. Pa. L. Rev. 165 (2016), 633.

[62] Jill Lepore. 2018. These truths: A history of the United States. WW Norton \& Company.

[63] Meira Levinson. 2012. No citizen left behind. Vol. 13. Harvard University Press.

[64] Zachary Lipton, Julian McAuley, and Alexandra Chouldechova. 2018. Does mitigating ML's impact disparity require treatment disparity?. In Advances in Neural Information Processing Systems. 8125-8135.

[65] Lydia T Liu, Sarah Dean, Esther Rolf, Max Simchowitz, and Moritz Hardt. 2018. Delayed impact of fair machine learning. In International Conference on Machine Learning.

[66] Catharine A MacKinnon. 2016. Substantive equality revisited: A reply to Sandra Fredman. International journal of constitutional law 14, 3 (2016), 739-746.

[67] Catharine A MacKinnon. 2017. Substantive equality revisited: A rejoinder to Sandra Fredman. International fournal of Constitutional Law 15, 4 (2017), $1174-1177$.

[68] Dee Masters. 2017. Identifying Direct Discrimination in 'Proxy Cases' after R (on the Application of Coll) v Secretary of State for Justice. Cloisters. https://www.cloisters.com/identifying-direct-discrimination-in-proxy-casesafter-r-on-the-application-of-coll-v-secretary-of-state-for-justice/ (2017).

[69] Aileen McColgan. 2014. Discrimination, equality and the law. Bloomsbury Publishing.

[70] Christopher McCrudden. 1982. Institutional discrimination. Oxford f. Legal Stud. 2 (1982), 303.

[71] Christopher McCrudden and Sacha Prechal. 2009. The Concepts of Equality and Non-discrimination in Europe: A practical approach. European Commission, Directorate-General for Employment, Social Affairs and Equal Opportunities, Unit G 2 (2009).

[72] Arthur S Miller and Ronald F Howell. 1960. The Myth of Neutrality in Constitutional Adjudication. The University of Chicago Law Review 27, 4 (1960), 661-695.

[73] Martha Minow. 1990. Making all the difference: Inclusion, exclusion, and American law. Cornell University Press. 
[74] Thomas Nagel. 1997. Introduction of equality and preferential treatment. A Philosophy (1997).

[75] Cathy O'Neil. 2019. Comment Regarding Docket No. FR-6111-P02. https://clinic.cyber.harvard.edu/files/2019/10/HUD-Rule-Comment-ONEIL10-18-2019-FINAL.pdf.

[76] Dino Pedreshi, Salvatore Ruggieri, and Franco Turini. 2008. Discriminationaware data mining. In Proceedings of the 14th ACM SIGKDD international conference on Knowledge discovery and data mining. 560-568.

[77] Juan C Perdomo, Tijana Zrnic, Celestine Mendler-Dünner, and Moritz Hardt. 2020. Performative prediction. arXiv preprint arXiv:2002.06673 (2020)

[78] Manish Raghavan, Solon Barocas, Jon Kleinberg, and Karen Levy. 2020. Mitigating bias in algorithmic hiring: Evaluating claims and practices. In Proceedings of the 2020 Conference on Fairness, Accountability, and Transparency. 469-481.

[79] John Roberts. 2007. Parents Involved in Community Schools v. Seattle School Dist.

[80] Piotr Sapiezynski, Avijit Gosh, Levi Kaplan, Alan Mislove, and Aaron Rieke. 2019. Algorithms that" Don't See Color": Comparing Biases in Lookalike and Special Ad Audiences. arXiv preprint arXiv:1912.07579 (2019).

[81] Marshall Cohen Thomas Nagel Thomas Scanlon, Ronald Dworkin, et al. 1977. Equality and preferential treatment. Princeton University Press.

[82] Frederick Schauer. 2018. On Treating Unlike Cases Alike.

[83] Andrew Selbst. 2019. A New HUD Rule Would Effectively Encourage Discrimination by Algorithm. https://slate.com/technology/2019/08/hud-disparate-impactdiscrimination-algorithm.html (2019).

[84] Andrew D Selbst, Danah Boyd, Sorelle A Friedler, Suresh Venkatasubramanian, and Janet Vertesi. 2019. Fairness and abstraction in sociotechnical systems. In Proceedings of the Conference on Fairness, Accountability, and Transparency. 59-68.

[85] Tommie Shelby. 2016. Dark ghettos: Injustice, dissent, and reform. Harvard University Press.

[86] Reva B Siegel. 2017. Blind Justice: Why the Court Refused to Accept Statistical Evidence of Discriminatory Purpose in McCleskey v. Kemp-And Some Pathways for Charge. Nw. UL Rev. 112 (2017), 1269.

[87] Reva B Siegel. 2018. The Constitutionalism of Disparate Impact-Court-Centered and Popular Pathways: a Comment on Owen Fiss's Brennan Lecture. Calif. L. Rev. 106 (2018), 2001.

[88] John David Skrentny. 2018. The ironies of affirmative action: Politics, culture, and justice in America. University of Chicago Press.
[89] Sonya Sotomayor. 2014. Schuette v. Coalition to Defend Affirmative Action.

[90] Cass R Sunstein. 1992. Neutrality in constitutional law (with special reference to pornography, abortion, and surrogacy). Columbia Law Review 92, 1 (1992), $1-52$.

[91] Beverly Daniel Tatum. 2017. Why are all the Black kids sitting together in the cafeteria?: And other conversations about race. Basic Books.

[92] Christa Tobler. 2008. Limits and potential of the concept of indirect discrimination. Office for Official Publications of the European Communities.

[93] Jon Truby. 2018. Fintech and the city: Sandbox 2.0 policy and regulatory reform proposals. International Review of Law, Computers \& Technology (2018), 1-33.

[94] Upturn. 2018. Brief as Amicus Curiae Supporting Petitioners. https://www. upturn.org/static/files/2018-11-16_Upturn_Facebook_Amicus.pdf.

[95] Berk Ustun, Yang Liu, and David Parkes. 2019. Fairness without harm: Decoupled classifiers with preference guarantees. In International Conference on Machine Learning. 6373-6382.

[96] Michael Veale, Reuben Binns, and Jef Ausloos. 2018. When data protection by design and data subject rights clash. International Data Privacy Law 8, 2 (2018), 105-123.

[97] Wolfgang Von Leyden. 1985. Aristotle on equality and justice: His political argument. Springer.

[98] Michael Walzer. 2008. Spheres of justice: A defense of pluralism and equality. Basic books.

[99] Alice Xiang. 2021. Reconciling legal and technical approaches to algorithmic bias. Tennessee Law Review 88, 3 (2021).

[100] Iris Marion Young. 2011. Justice and the Politics of Difference. Princeton University Press.

[101] Muhammad Bilal Zafar, Isabel Valera, Manuel Gomez Rodriguez, and Krishna P Gummadi. 2017. Fairness beyond disparate treatment \& disparate impact: Learning classification without disparate mistreatment. In Proceedings of the 26th international conference on world wide web. 1171-1180.

[102] Muhammad Bilal Zafar, Isabel Valera, Manuel Rodriguez, Krishna Gummadi, and Adrian Weller. 2017. From parity to preference-based notions of fairness in classification. In Advances in Neural Information Processing Systems. 229-239.

[103] Muhammad Bilal Zafar, Isabel Valera, Manuel Gomez Rogriguez, and Krishna P Gummadi. 2017. Fairness constraints: Mechanisms for fair classification. In Artificial Intelligence and Statistics. PMLR, 962-970. 